\title{
Global sagittal axis: a step toward full-body assessment of sagittal plane deformity in the human body
}

\author{
Bassel G. Diebo, MD, Jonathan H. Oren, MD, Vincent Challier, MD, Renaud Lafage, MS, \\ Emmanuelle Ferrero, MD, Shian Liu, MD, Shaleen Vira, MD, Matthew Adam Spiegel, BA, \\ Bradley Yates Harris, JD, Barthelemy Liabaud, MD, Jensen K. Henry, BA, Thomas J. Errico, MD, \\ Frank J. Schwab, MD, and Virginie Lafage, PhD
}

Department of Orthopaedic Surgery, New York University Langone Medical Center, New York, New York

\begin{abstract}
OBJECTIVE Sagittal malalignment requires higher energy expenditure to maintain an erect posture. Because the clinical impact of sagittal alignment is affected by both the severity of the deformity and recruitment of compensatory mechanisms, it is important to investigate new parameters that reflect both disability level and compensatory mechanisms for all patients. This study investigated the clinical relevance of the global sagittal axis (GSA), a novel measure to evaluate the standing axis of the human body.

METHODS This is a retrospective review of patients who underwent full-body radiographs and completed health-related quality of life (HRQOL) questionnaires: Oswestry Disability Index (ODI), Scoliosis Research Society-22, EuroQol-5D (EQ-5D), and the visual analog scale for back and leg pain. The GSA was defined as the angle formed by a line from the midpoint of the femoral condyles to the center of $\mathrm{C}-7$, and a line from the midpoint between the femoral condyles to the posterior superior corner of the S-1 sacral endplate. After evaluating the correlation of GSA/HRQOL with sagittal parameters, linear regression models were generated to investigate how ODI and GSA related to radiographic parameters (T-1 pelvic angle, pelvic retroversion, knee flexion, and pelvic posterior translation).
\end{abstract}

RESULTS One hundred forty-three patients (mean age 44 years) were included. The GSA correlated significantly with all $H R Q O L$ (up to $r=0.6$ with EQ-5D) and radiographic parameters (up to $r=0.962$ with sagittal vertical axis). Regression between ODI and sagittal radiographic parameters identified the GSA as an independent predictor $\left(r=0.517, r^{2}=\right.$ $0.267 ; p<0.001$ ). Analysis of standardized coefficients revealed that when controlling for deformity, the GSA increased with a concurrent decrease in pelvic retroversion $(-0.837)$ and increases in knee flexion $(+0.287)$ and pelvic posterior translation (+0.193).

CONCLUSIONS The GSA is a simple, novel measure to assess the standing axis of the human body in the sagittal plane. The GSA correlated highly with spinopelvic and lower-extremities sagittal parameters and exhibited remarkable correlations with $\mathrm{HRQOL}$, which exceeded other commonly used parameters.

http://thejns.org/doi/abs/10.3171/2016.2.SPINE151311

KEY WORDS full-body sagittal alignment; global sagittal axis; GSA; deformity

$\mathrm{T}$ HE radiographic analysis of the sagittal plane has become a critical part of the clinical evaluation and surgical care of spine patients. Much of the current knowledge of the sagittal plane can be traced to the work of Jean Dubousset and his theory of the conus of economy. According to Dubousset, the normal human posture assumes a stance limited to a narrow anterior-posterior range to minimize muscle exertion. ${ }^{3}$ Following Dubousset's work, an era of sagittal plane research unfolded as various fields investigated sagittal plane deformities in patients. .,4,7-9,11-15

Studies have shown that the loss of lumbar lordosis is the common driver of sagittal spinal deformity, resulting in the loss of physiological and functional alignment of the spine. ${ }^{5}$ However, these consequences extend beyond the

ABBREVIATIONS CBVA = chin-brow vertical angle; EQ-5D = EuroQol-5D; GSA = global sagittal axis; $\mathrm{HRQOL}=$ health-related quality of life; $\mathrm{KA}=\mathrm{knee}$ flexion angle; $\mathrm{LL}=$ lumbar lordosis; ODI = Oswestry Disability Index; PI = pelvic incidence; PT = pelvic tilt; SRS22r = Scoliosis Research Society-22, refined; SVA = sagittal vertical axis; TPA = T-1 pelvic angle; VAS = visual analog scale.

SUBMITTED November 3, 2015. ACCEPTED February 26, 2016.

INCLUDE WHEN CITING Published online May 20, 2016; DOI: 10.3171/2016.2.SPINE151311. 
spinal column, as a cascade of compensatory mechanisms is recruited to counteract spinal sagittal malalignment. ${ }^{2}$ Mechanisms such as thoracic hypokyphosis, hip extension (pelvic retroversion around the hip joint), and increased flexion of the knee and ankle are commonly recruited..$^{10,16}$

The combination of sagittal spinal deformity and pelvic/lower limb compensation results in an alignment in which the trunk is tilted anteriorly while the pelvis translates posterior to the gravity line. ${ }^{17}$ These phenomena function to counteract the sagittal deformity by keeping the center of gravity over a narrow area between the feet. ${ }^{3}$ Many radiographic parameters must be analyzed to characterize the most extreme states of deformity, in which pelvic and subpelvic components are involved in compensation.

Lafage et al. ${ }^{10}$ found that the inability to recruit compensatory mechanisms worsens the clinical impact of sagittal deformity. They demonstrated that there is a subset of patients with sagittal malalignment in whom recruitment of pelvic retroversion failed; these patients were subsequently more disabled than those in whom the pelvis was able to compensate. However, these results were not investigated using full-standing radiographs, and thus were unable to unmask additional lower-extremity compensatory mechanisms.

In the era of advanced imaging techniques, it is important to investigate new parameters to reflect various levels of deformity. These parameters may provide the ability to distinguish between patients with similar deformities but different methods of compensation. Finally, given the time constraints in a busy clinical practice, a simple measure may be beneficial as a screening tool to provide rapid assessment of the global sagittal plane without extensive analysis of multiple parameters. This study investigated the clinical relevance of the global sagittal axis (GSA). The authors hypothesized that the GSA would significantly correlate with regional radiographic parameters along the full-body axis, as well as clinical outcome scores.

\section{Methods \\ Study Design}

This institutional review board-approved study is a single-center retrospective review of patients who underwent full-body stereoradiography for adult spinal deformity based on their chart diagnoses between 2012 and 2014 . For inclusion, patients must have completed their healthrelated quality of life (HRQOL) questionnaires within 1 week (before or after) of clinical consultation and radiographic imaging. Patients with fractures, malignancies, infections, neuromuscular scoliosis, ankylosing spondylitis, or diffuse idiopathic skeletal hyperostosis were excluded.

\section{Data Collection and Radiographic Analysis}

Data collected consisted of basic demographic information including age, sex, body mass index, and history of previous spine surgery, as well as the following HRQOL questionnaires: Oswestry Disability Index (ODI), Scoliosis Research Society-22, refined (SRS22r), EuroQol-5D (EQ-5D), and visual analog scale (VAS) for back and leg pain.
Full-body radiographic analysis was performed using Surgimap (Nemaris, Inc.), a validated software. ${ }^{19}$ Spinopelvic radiographic parameters included pelvic incidence (PI), lumbar lordosis (LL), pelvic tilt (PT), C7-S1 sagittal vertical axis (SVA), and T-1 pelvic angle (TPA). Lowerextremity radiographic parameters included knee flexion angle (KA), ankle flexion angle, and pelvic shift. Craniocervical radiographic parameters included $\mathrm{C} 2-7$ cervical curvature, C2-7 SVA, and chin-brow vertical angle (CBVA). The GSA was defined as the angle formed by a line from the midpoint of the 2 distal femoral condyles to the center of C-7, and a line from the midpoint between the 2 distal femoral condyles to the posterior superior corner of the S-1 sacral endplate (Fig. 1).

\section{Clinical Outcomes Analysis}

The mean values, SDs, and ranges for all radiographic parameters were described. Pearson correlation was performed to investigate the relationship between the GSA and full-body radiographic parameters, as well as between radiographic parameters and HRQOL. A linear regression model was used, with GSA as the dependent variable and PT as an independent variable, controlling for global deformity (measured by TPA), pelvic morphology (measured by PI), and sex. Then, the same model was performed, but $\mathrm{KA}$ and pelvic shift were added as independent variables. A linear regression model was again performed to predict ODI scores based on GSA values, and thresholds of GSA corresponding to ODI values of 20,30 , and 40 were identified.

\section{Results \\ Patient Population}

One hundred forty-three patients met inclusion criteria, all with diagnoses of degenerative lumbar scoliosis, progressive idiopathic scoliosis, or kyphoscoliosis. The mean age of the cohort was $44.1 \pm 22.1$ years; $46.8 \%$ of patients were younger than 45 years, $30.8 \%$ of patients were between 45 and 65 years, and $22.3 \%$ were older than 65 years. Seventy-eight percent of patients were women, and the mean body mass index was $24.3 \mathrm{~kg} / \mathrm{m}^{2}$. Sixty-five percent of patients had no history of spine surgery. Patients had the following HRQOL mean scores: ODI $24.8 \pm 22.3$, range 0-100; SRS22r $3.4 \pm 0.7$, range 1.7-4.7; EQ-5D 7.8 \pm 2.2 , range 5-14; VAS back $4.8 \pm 3.3$, range $0-10$; and VAS leg $2.5 \pm 3.4$, range $0-10$.

\section{Radiographic Measurements}

The mean GSA was $0.7^{\circ} \pm 5.9^{\circ}$ (range $-8.2^{\circ}$ to $19.5^{\circ}$ ) The cohort had a mean PI of $53.6^{\circ} \pm 13.9^{\circ}$ (range $21.5^{\circ}-$ $103.3^{\circ}$ ), a mean PI-LL of $4.4^{\circ} \pm 23.2^{\circ}$ (range $-63.8^{\circ}$ to $66.5^{\circ}$ ), a mean PT of $19.0^{\circ} \pm 13.1^{\circ}$ (range $-34.1^{\circ}$ to $50.0^{\circ}$ ), a mean TPA of $14.9^{\circ} \pm 14.9^{\circ}$ (range $-30.4^{\circ}$ to $50.8^{\circ}$ ), and a mean SVA of $17.2 \pm 55.4 \mathrm{~mm}$ (range $-71.2^{\circ}$ to $200.4^{\circ}$ ). The mean values for lower-limb measurements were as follows: KA $3.0^{\circ} \pm 9.0^{\circ}$ (range $-15.1^{\circ}$ to $42.8^{\circ}$ ), ankle dorsiflexion $6.5^{\circ} \pm 4.1^{\circ}$ (range $-2.8^{\circ}$ to $23.3^{\circ}$ ), and pelvic posterior shift $1.4 \pm 41.7 \mathrm{~mm}\left(\right.$ range $-109.8^{\circ}$ to $\left.117.9^{\circ}\right)$. The cohort had the following mean values and ranges for cervical parameters: $\mathrm{C} 2-7$ cervical curvature $8.3^{\circ} \pm 23.6^{\circ}$ (range $-35.7^{\circ}$ 


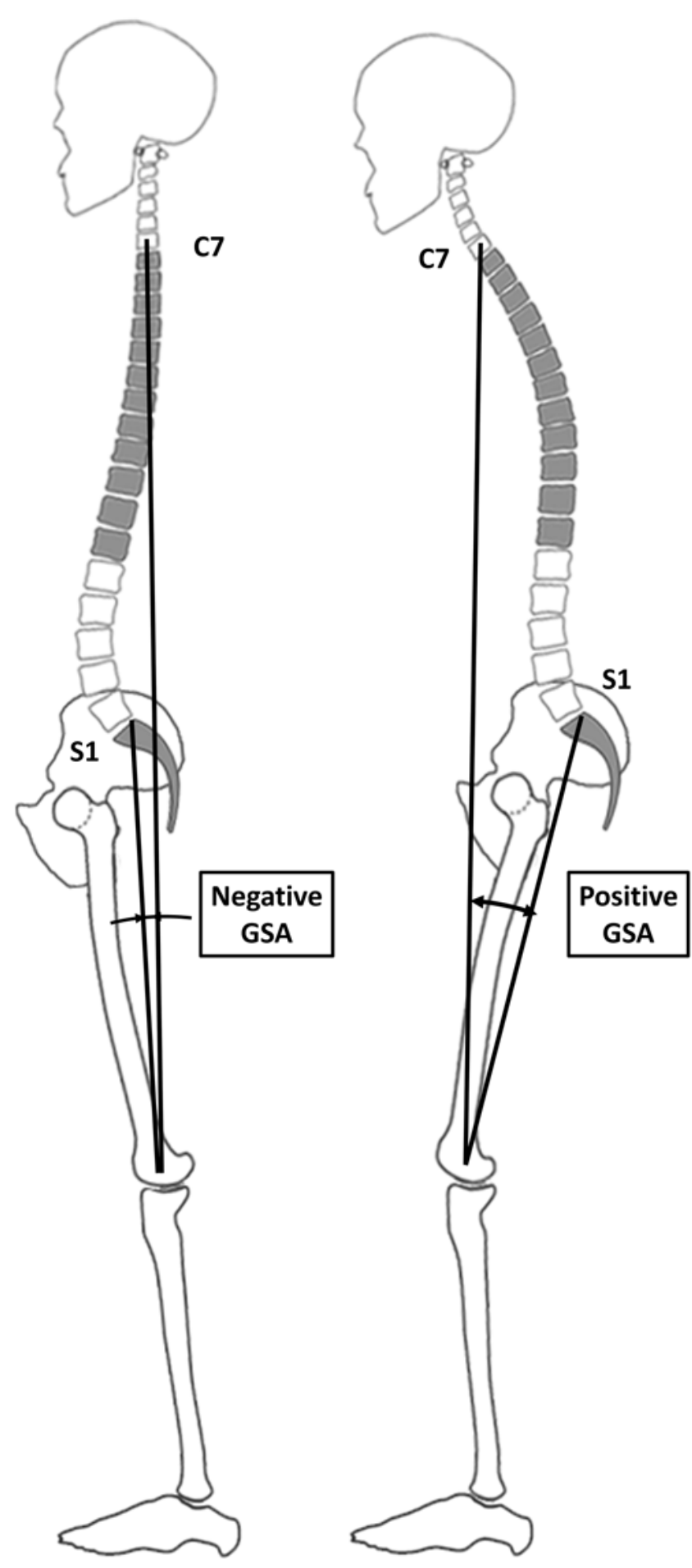

FIG. 1. Illustrations showing the GSA: negative value (left) and positive value (right).

to $146.2^{\circ}$ ), $\mathrm{C} 2-7 \mathrm{SVA} 17.2 \mathrm{~mm} \pm 55.4 \mathrm{~mm}$ (range $-90.8^{\circ}$ to $82.4^{\circ}$ ), and $\mathrm{CBVA} 6.7^{\circ} \pm 13.2^{\circ}$ (range $-16.1^{\circ}$ to $87.7^{\circ}$ ).

\section{Correlation Analysis}

The GSA significantly correlated with the classic SRSSchwab spinopelvic sagittal modifiers (PI-LL, PT, and
SVA), as well as lower-limb and craniocervical parameters. Correlation coefficients are reported in Table 1; all correlations were significant $(\mathrm{p}<0.05)$.

The GSA significantly correlated with all HRQOL scores. The GSA had better correlations with ODI, SRS22r, EQ-5D, and VAS leg pain scores than with any of the other radiographic parameters. After SVA, GSA was the second most correlated parameter with VAS back pain. Correlation coefficients are reported in Table 2.

\section{Regression Analysis}

Analysis of standardized coefficients of the regression analysis between the GSA and other sagittal spinopelvic parameters revealed that when controlling for deformity (TPA), PI, and sex, the GSA increased (beta +1.991) with a concurrent decrease in PT (-1.232). These findings echoed those of ODI, EQ-5D, VAS back/leg pain, and SRS22r (Table 3).

Adding KA and pelvic shift as independent variables to the regression analysis revealed an increase in the GSA with a concurrent increase of KA and pelvic shift, reflected by standardized coefficients of +0.287 and +0.193 , respectively. The TPA and PT standardized coefficients in this model were +1.324 and -0.837 , respectively: $r=0.996$.

Regression between ODI, classic parameters (SVA, PT, and PI-LL), and lower-limb parameters retained the GSA as an independent predictor $\left(r=0.517, \mathrm{r}^{2}=0.267\right.$; $\mathrm{p}<0.001)$. Greater GSA values were associated with increased scores of disability: $-1.5^{\circ}$ for ODI $=20,4^{\circ}$ for ODI $=30$, and $9^{\circ}$ for ODI $=40$.

\section{Discussion}

\section{Clinical Relevance of the GSA}

The present study introduced the GSA, a simple and novel measurement that correlates with established sagittal spinopelvic radiographic parameters. Moreover, the GSA expands our understanding of the spine and pelvis to include the full-body axis. The GSA is sensitive to spine, pelvic, and lower-extremity compensatory changes in the sagittal plane and holds one of the strongest correlations with patient-reported clinical scores reported in the literature (0.6 for EQ-5D). The advantage of the GSA is that it

TABLE 1. Correlation coefficients between GSA and full-body sagittal radiographic parameters*

\begin{tabular}{cc}
\hline Sagittal Radiographic Measure & GSA \\
\hline PT & 0.622 \\
\hline PI minus LL & 0.771 \\
\hline TPA & 0.844 \\
\hline SVA & 0.962 \\
\hline KA & 0.793 \\
\hline Ankle dorsiflexion & 0.561 \\
\hline Pelvic posterior shift & 0.870 \\
\hline C2-7 cervical curvature & 0.326 \\
\hline C2-7 SVA & 0.216 \\
\hline CBVA & -0.251 \\
\hline
\end{tabular}

* All correlations were significant $(p<0.05)$. 
TABLE 2. Correlation coefficients between GSA, full-body sagittal radiographic parameters, and HRQOL measures*

\begin{tabular}{lccccc}
\hline $\begin{array}{c}\text { Sagittal Radiographic } \\
\text { Measure }\end{array}$ & ODI & SRS22r & EQ-5D & $\begin{array}{c}\text { VAS } \\
\text { Back }\end{array}$ & $\begin{array}{c}\text { VAS } \\
\text { Leg }\end{array}$ \\
\hline GSA & $\mathbf{0 . 5 1 7}$ & $-\mathbf{0 . 5 4 3}$ & $\mathbf{0 . 5 9 4}$ & 0.371 & $\mathbf{0 . 4 5 2}$ \\
\hline PT & 0.269 & -0.260 & 0.387 & - & 0.205 \\
\hline PI minus LL & 0.336 & -0.344 & 0.440 & 0.256 & 0.339 \\
\hline TPA & 0.392 & -0.397 & 0.509 & 0.260 & 0.335 \\
\hline SVA & 0.505 & -0.534 & 0.575 & $\mathbf{0 . 3 9 9}$ & 0.448 \\
\hline KA & 0.508 & -0.510 & 0.552 & 0.337 & 0.387 \\
\hline Ankle dorsiflexion & 0.485 & -0.441 & 0.523 & 0.299 & 0.330 \\
\hline Pelvic posterior shift & 0.392 & -0.443 & 0.459 & 0.265 & 0.357 \\
\hline
\end{tabular}

$-=$ no significant correlation.

* All correlations were significant $(p<0.05)$. The highest correlation coef-

ficient in each column is in boldface type.

reflects different stages of deformity more accurately than previously defined spinopelvic parameters. Specifically, the GSA becomes a critically important alignment parameter in assessing 2 types of patients: those whose spine is severely deformed and subsequently recruit lower-limb compensations after pelvic retroversion is exhausted; and those who do not have the ability to compensate by pelvic retroversion. The GSA is able to reflect the disability of these 2 populations because of the involvement of lower limbs in the sagittal profile.

Researchers have attempted to deconstruct the complex system of the sagittal plane into regional radiographic parameters to evaluate different musculoskeletal components. ${ }^{7,10,18}$ Studies by both Dubousset and Duval-Beaupère et al. highlighted the importance of incorporating the pelvis in the assessment of spinal malalignment. ${ }^{3,4}$ The pelvis provides a highly mobile intercalated segment between the trunk and lower limbs that is mediated through the hip joint. Although the pelvis has been extensively studied in the spinal literature, the lower limbs are beginning to be investigated using full-body radiographic images. Lowerextremity compensation via increased flexion of the knees and ankles and subsequent pelvic shift plays a significant role in attempts at sagittal realignment and is therefore the direct effect of a pathological spinal deformity. 2,6,16,17

Measurement of multiple radiographic spinopelvic parameters in a patient with deformity offers insight into the relationship between deformity and compensation. However, performing these analyses could be cumbersome for clinicians, because they require time and experience with multiple parameters. The GSA offers a simple and efficient method to capture clinically relevant information on standing alignment and to understand the implied disability. Indeed, the value of the GSA as a simple method to assess standing sagittal alignment should support its use as a screening tool. Once the deformity is identified, more detailed traditional analysis would obviously be required to understand the etiology of the pathology as well as the surgical plan.

\section{The Compensation Cascade}

Compensation for spinal malalignment by pelvic retro-
TABLE 3. Regression model to predict GSA and clinical scores based on PT while controlling for deformity (represented by TPA measure), PI, and patient sex

\begin{tabular}{|c|c|c|c|c|c|}
\hline \multirow{2}{*}{$\begin{array}{c}\text { Dependent } \\
\text { Variable }\end{array}$} & \multicolumn{2}{|c|}{ Standardized Coefficient } & \multicolumn{2}{|c|}{ Standard Error } & \multirow[b]{2}{*}{$r$} \\
\hline & TPA & PT & TPA & PT & \\
\hline GSA & 1.991 & -1.232 & 0.027 & 0.031 & 0.956 \\
\hline ODI & 1.038 & -0.696 & 0.301 & 0.343 & 0.470 \\
\hline$E Q-5 D$ & 1.083 & -0.619 & 0.028 & 0.032 & 0.558 \\
\hline VAS back & 0.989 & -0.785 & 0.047 & 0.053 & 0.390 \\
\hline VAS leg & 1.057 & -0.777 & 0.047 & 0.053 & 0.442 \\
\hline SRS22r & -1.200 & 0.862 & 0.010 & 0.011 & 0.503 \\
\hline
\end{tabular}

version around the femoral heads reaches a limit as maximal hip extension is achieved. When greater compensation is needed or when hip pathology limits compensation, the lower extremities increase flexion of the knees and ankles. This coupled motion offers a patient the ability to translate the pelvis posteriorly with respect to the feet. In turn, such a maneuver regulates the anterior truncal shift caused by sagittal malalignment and maintains the gravity line projection between the feet. It is at this point that the GSA, which is anchored at the knee, demonstrates significant correlation with patient-reported clinical outcome scores, demonstrating the clinical relevance of the knees in the consideration of sagittal plane stance. Although the GSA does not directly include ankle measurement, we hypothesized that the compensatory actions of the knee and ankle are intricately linked and usually occur concurrently. Therefore, the GSA may represent the total contributions of the knees and ankles in the compensation for sagittal malalignment.

\section{Radiographic Parameters, HRQOL Scores, and the Gap}

The literature is replete with radiographic parameters that increase with malalignment, compensation, or both. However, patients in whom there is a failure to recruit pelvic retroversion to counteract their spinal malalignment have been shown to be more disabled..$^{10}$ Ironically, the radiographic analysis of this subset of patients using 1 angular measure often reveals less severe deformity compared with patients with compensated alignment (Fig. 2). Thus, 2 parameters are used to distinguish among patients with similar malalignment (e.g., SVA) and different compensation (e.g., PT).

In this study, the regression analysis showed that after controlling for deformity, the GSA increases with decreased pelvic compensation. These findings were also observed for clinical outcomes scores. It is possible that these patients bypass pelvic compensation because of gluteal weakness, fatigue, or hip pathology, and instead compensate with their lower limbs, manifesting in an increased GSA. Whereas 1 traditional spinopelvic parameter may underestimate the deformity in these patients, the GSA captures the effects of the pelvis and subpelvis compensatory mechanisms.

\section{The Musculoskeletal System is 1 Unit}

This study strives to illustrate the importance and utility 

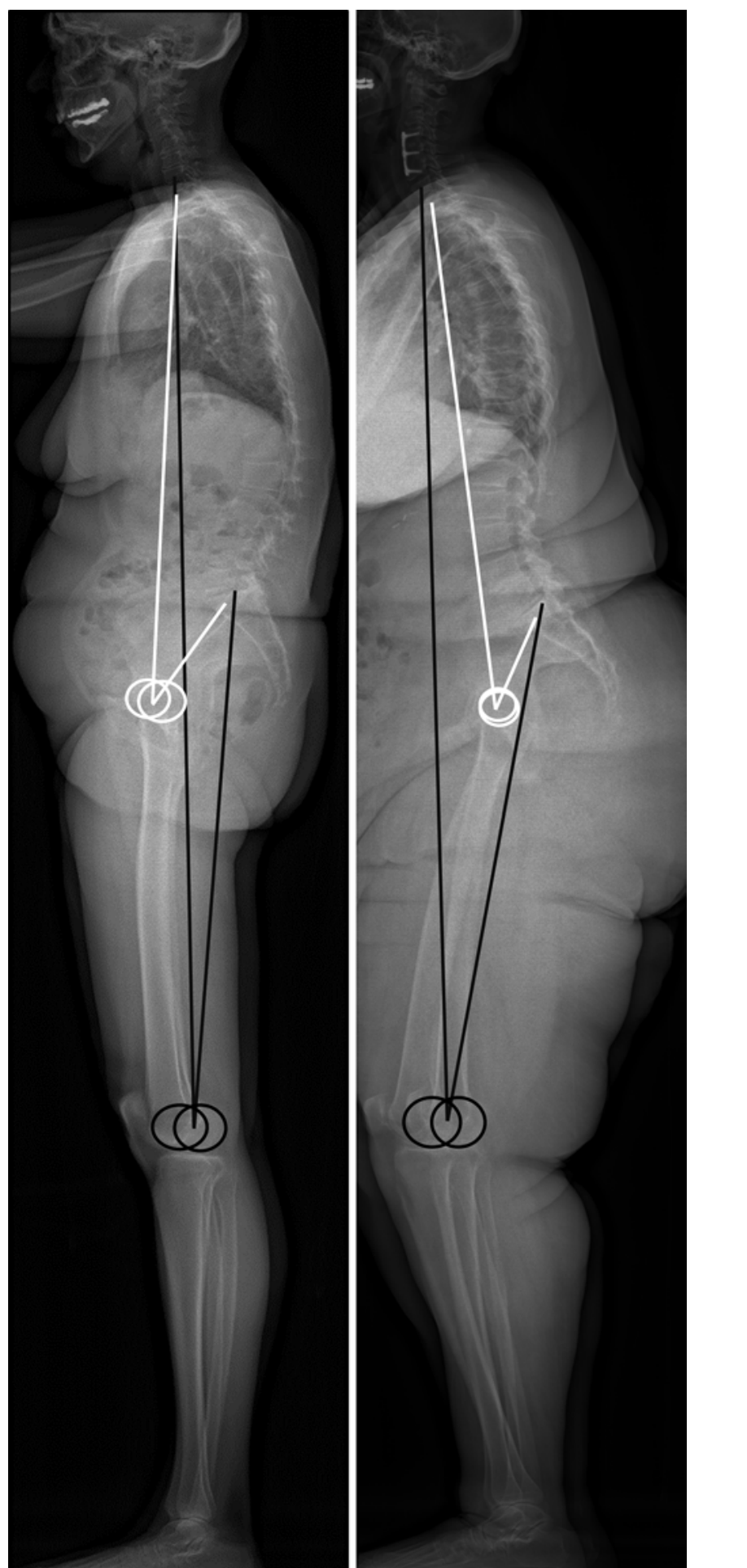

the skeletal components, chiefly the hip and knee joints, is vital. Chronic pathology such as sagittal deformity, which may occur over decades of degenerative changes, tends to engage several components of the standing axis. Clinically, primary pathologies of the spine, hips, and knees often present simultaneously in patients. In patients with a single or bilateral joint complaint, the GSA may assist in early recognition of a more severe underlying problem.

The GSA offers a view of global standing ability, which is supported by strong statistical relationships with each regional parameter along the full-body axis. Full-body sagittal malalignment is a problem that incorporates each component of the musculoskeletal system. The GSA is a parameter that may help surgeons efficiently evaluate the interplay between the spine, pelvis, and lower limbs in the sagittal plane.

\section{Limitations and Future Work}

The GSA evaluates static postural alignment. One should always remember that radiographs are bone shadows and are merely 1 tool to understand the comprehensive picture of the deformity. A snapshot analysis with 1 parameter has inherent limitations, e.g., the current data were compiled from the initial clinic visit for every patient in the study, and thus represent a patient population at a specific point in their clinical pathology. Understanding the patient's deformity in the overall context and evolution of his or her disease is crucial for treatment. Further studies should investigate the GSA concept with 2 or more time points as well as the relevance of the GSA in other populations, including those with lower-limb joint pathologies. Finally, the availability of full-body imaging, and therefore any lower-limb parameters, is still limited to centers with dedicated imaging technology or to research institutions with interest in the full-body sagittal profile. However, as the understanding of musculoskeletal deformity progresses, the use of full-body imaging for patients may become more widespread.

\section{Conclusions}

The GSA is a simple, novel measure to assess the sagittal standing axis of the human body. The GSA is highly correlated with spinal, pelvic, and lower-extremity sagittal parameters and exhibited remarkable correlations with patient-reported clinical scores, exceeding many other parameters commonly reported in the literature. In both severe sagittal deformity and the absence of pelvic compensation, the GSA is able to represent the involvement of the lower limbs. Moreover, the GSA is a strong indicator of patient disability and clinical scores. The GSA provides a common, efficacious parameter by which health care providers can communicate the deterioration of standing alignment while addressing a regional sagittal deformity anywhere from the cervical region to the ankles.

\section{References}

1. Baek SW, Kim C, Chang H: The relationship between the spinopelvic balance and the incidence of adjacent vertebral fractures following percutaneous vertebroplasty. Osteoporos Int 26:1507-1513, 2015 
2. Diebo BG, Ferrero E, Lafage R, Challier V, Liabaud B, Liu $\mathrm{S}$, et al: Recruitment of compensatory mechanisms in sagittal spinal malalignment is age and regional deformity dependent: a full-standing axis analysis of key radiographical parameters. Spine (Phila Pa 1976) 40:642-649, 2015

3. Dubousset J: Three-dimensional analysis of the scoliotic deformity, in Weinstein SL (ed): The Pediatric Spine: Principles and Practices. New York: Raven Press, pp 479-496, 1994

4. Duval-Beaupère G, Schmidt C, Cosson P: A barycentremetric study of the sagittal shape of spine and pelvis: the conditions required for an economic standing position. Ann Biomed Eng 20:451-462, 1992

5. Farcy JP, Schwab FJ: Management of flatback and related kyphotic decompensation syndromes. Spine (Phila Pa 1976) 22:2452-2457, 1997

6. Glassman SD, Bridwell K, Dimar JR, Horton W, Berven S, Schwab F: The impact of positive sagittal balance in adult spinal deformity. Spine (Phila Pa 1976) 30:2024-2029, 2005

7. Iida T, Suzuki N, Kono K, Ohyama Y, Imura J, Ato A, et al: Minimum 20 years long-term clinical outcome after spinal fusion and instrumentation for scoliosis. Spine (Phila Pa 1976) 40:E922-E928, 2015

8. Jentzsch T, Geiger J, König MA, Werner CM: Hyperlordosis is associated with facet joint pathology at the lower lumbar spine. J Spinal Disord Tech [epub ahead of print], 2013

9. Kumar MN, Baklanov A, Chopin D: Correlation between sagittal plane changes and adjacent segment degeneration following lumbar spine fusion. Eur Spine J 10:314-319, 2001

10. Lafage V, Schwab F, Patel A, Hawkinson N, Farcy JP: Pelvic tilt and truncal inclination: two key radiographic parameters in the setting of adults with spinal deformity. Spine (Phila Pa 1976) 34:E599-E606, 2009

11. Lazennec JY, Brusson A, Rousseau MA: THA patients in standing and sitting positions: a prospective evaluation using the low-dose "full-body" EOS imaging system. Semin Arthroplasty 23:220-225, 2012

12. Lazennec JY, Charlot N, Gorin M, Roger B, Arafati N, Bissery A, et al: Hip-spine relationship: a radio-anatomical study for optimization in acetabular cup positioning. Surg Radiol Anat 26:136-144, 2004

13. Liu S, Ferrero E, Liabaud B, Challier V, Lafage R, Diebo BG, et al: Global sagittal alignment analysis including lower extremities: role of pelvic translation and the lower extremities in compensation to spinal deformity. Spine J 14 Suppl:S138, 2014

14. Miyakoshi N, Hongo M, Kobayashi T, Abe T, Abe E, Shimada Y: Improvement of spinal alignment and quality of life after corrective surgery for spinal kyphosis in patients with osteoporosis: a comparative study with non-operated patients. Osteoporos Int 26:2657-2664, 2015
15. Murata Y, Takahashi K, Yamagata M, Hanaoka E, Moriya $\mathrm{H}$ : The knee-spine syndrome. Association between lumbar lordosis and extension of the knee. J Bone Joint Surg Br 85:95-99, 2003

16. Obeid I, Hauger O, Aunoble S, Bourghli A, Pellet N, Vital JM: Global analysis of sagittal spinal alignment in major deformities: correlation between lack of lumbar lordosis and flexion of the knee. Eur Spine J 20 (Suppl 5):681-685, 2011

17. Schwab F, Lafage V, Boyce R, Skalli W, Farcy JP: Gravity line analysis in adult volunteers: age-related correlation with spinal parameters, pelvic parameters, and foot position. Spine (Phila Pa 1976) 31:E959-E967, 2006

18. Schwab F, Patel A, Ungar B, Farcy JP, Lafage V: Adult spinal deformity-postoperative standing imbalance: how much can you tolerate? An overview of key parameters in assessing alignment and planning corrective surgery. Spine (Phila Pa 1976) 35:2224-2231, 2010

19. Vila-Casademunt A, Pellisé F, Acaroglu E, Pérez-Grueso FJS, Martín-Buitrago MP, Sanli T, et al: The reliability of sagittal pelvic parameters: the effect of lumbosacral instrumentation and measurement experience. Spine (Phila Pa 1976) 40:E253-E258, 2015

\section{Disclosures}

Dr. Schwab has direct stock ownership in Nemaris, Inc., and is a consultant for K2M, Medicrea, NuVasive, Zimmer-Biomet, and MSD. He has speaking/teaching arrangements with K2M, Medicrea, NuVasive, Zimmer-Biomet, and MSD. Dr. Schwab has received clinical or research support from SRS, AO, DePuy Spine, and Syntesis. He is a patent holder for MSD and K2M. Dr. Lafage has direct stock ownership in Nemaris, Inc., and is a consultant for NuVasive. Dr. Lafage has received clinical or research support from SRS, NIH, and DePuy Spine Synthesis. She has speaking/teaching arrangements with Medicrea, NuVasive, DePuy Spine, and Nemaris, Inc.

\section{Author Contributions}

Conception and design: Diebo, Challier. Acquisition of data: $\mathrm{R}$ Lafage. Analysis and interpretation of data: Diebo. Drafting the article: Diebo, Oren, Challier, Ferrero, Liu, Vira, Spiegel, Harris, Liabaud, Henry, Errico, Schwab, V Lafage. Statistical analysis: Diebo. Study supervision: Schwab, V Lafage.

\section{Correspondence}

Bassel G. Diebo, Department of Orthopaedic Surgery, NYU Langone Medical Center, 306 East 15th St., New York, NY 10003. email: dr.basseldiebo@gmail.com. 